\title{
Correction to: What Is Random?
}

\section{Correction to: \\ E. Beltrami, What Is Random?, https://doi.org/10.1007/978-1- 0716-0799-2}

This book was inadvertently published with typesetting errors. Fonts were applied inconsistently to mathematical content, resulting in variations in the appearance of some letters and symbols which may have obscured mathematical meaning. All characters that were incorrectly typeset have been corrected in the present version.

The updated online version of this book can be found at https://doi.org/10.1007/978-1-0716-0799-2 